\title{
COPD - eine chronische Krankheit auf dem Vormarsch
}

Otto Brändli,

Erich W. Russi

1 GOLD: Global Strategy for the Diagnosis, Management and Prevention of COPD. Global Initiative for Chronic Obstructive Lung Disease 2007. www.goldcopd.org.

2 Bridevaux PO, Gerbase MW, Probst-Hensch NM, Schindler C, Gaspoz JM, Rochat T. Long-term decline in lung function, utilisation of care and quality of life in modified GOLD stage 1 COPD. Thorax. 2008;63:768-74.

3 Lundbäck B. Not $15 \%$ but $50 \%$ of smokers develop COPD? Resp Medicine. 2003;97:115-22.

Korrespondenzen:

Prof. Dr. med. Erich W. Russi Klinik für Pneumologie

Universitätsspital

Rämistrasse 100

CH-8091 Zürich

erich.russi@usz.ch

Dr. med. Otto Brändli

Hömelstrasse 15

CH-8636 Wald

braendli@swisslung.org
Im Gegensatz zu anderen chronischen Krankheiten ist die Chronisch Obstruktive Lungenkrankheit COPD weiter im Zunehmen begriffen. Weltweit hat sie heute Rang vier der häufigsten Todesursachen erreicht. Obwohl die COPD immer noch zu den unheilbaren Erkrankungen zählt, gibt es wichtige Fortschritte in ihrer Behandlung. Therapeutischer Nihilismus ist deshalb fehl am Platz.

Neueste Daten zur Häufigkeit in der Schweiz sind erschreckend und stehen in krassem Gegensatz zur Bekanntheit dieses gesundheitlich gravierenden Problems und den im Vergleich mit deutlich selteneren Krankheiten bescheidenen Ausgaben für die Forschung. Nicht nur in der Bevölkerung und bei Rauchern, auch im Praxisalltag ist das Bewusstsein für diese Erkrankung und ihre Prävention noch zu gering. Moderne Behandlungskonzepte und die weiter steigende Zahl von Betroffenen, vor allem Frauen, werden die Kosten in diesem Bereich ansteigen lassen. Dies ist um so bedauerlicher, als die Häufigkeit der COPD durch geeignete Präventionsmassnahmen, allen voran den Kampf gegen das Zigarettenrauchen, reduziert werden könnte.

\section{Das Krankheitsbild der COPD}

Die COPD ist kein einheitliches Krankheitsbild. Sie wurde ursprünglich von den Engländern als schleichend verlaufende chronische Bronchitis, die mit Husten, Auswurf und zunehmender Atemnot einhergeht, charakterisiert, von den Amerikanern pauschal als Emphysem bezeichnet. Seit Jahren wird die COPD durch eine mittels Spirometrie diagnostizierbare, nicht reversible Behinderung des Atemflusses definiert [1] (Abb. 1). Der Quotient $\left(\mathrm{FEV}_{1} / \mathrm{FVC}\right)$ zwischen Erstsekundenvolumen $\left(\mathrm{FEV}_{1}\right)$ und forcierter Vitalkapazität (FVC) ist permanent pathologisch tief $(<0,70)$. Der Schweregrad der Erkrankung (Stadium I-IV nach GOLD) wird durch den prozentualen Sollwert des $\mathrm{FEV}_{1}$ abgeschätzt. Nicht jeder Patient mit Symptomen einer chronischen Bronchitis (Husten, Auswurf >3 Monate während zweier konsekutiver Jahre) leidet an einer COPD und nicht jeder Patient mit einer COPD klagt über Symptome einer chronischen Bronchitis. Eine gute Korrelation besteht aber zwischen dem Ausmass der Atemnot bei körperlicher Belastung und Einschränkung der Lungenfunktion.
Die COPD ist nach heutigem Verständnis eine Systemerkrankung. Im Zentrum steht die chronische Entzündung der Lunge, die sich systemisch auswirkt. Diese mottende Inflammation, die mit einem erhöhten CRP einhergeht ist auch ein Risikofaktor für kardiovaskuläre Erkrankungen und mitverantwortlich für die Muskelschwäche und das Untergewicht vieler COPD-Patienten. Die COPD wird meist erst spät diagnostiziert. Häufig ist dann die Lungenfunktion bereits unter $50 \%$ abgesunken und die Lebenserwartung deutlich reduziert.

\section{Die Epidemiologie in der Schweiz}

Genaue Morbiditätsdaten über COPD gibt es leider für die Schweiz nicht. Die vom Bundesamt für Statistik ausgewerteten Todesfallbescheinigungen zeigen einen Anstieg der Erst- und Nebendiagnosen zwischen 1969 und 2003 um 81\% auf insgesamt gegen 4000 Todesfälle pro Jahr.

Die über 11 Jahre beobachtete, zufällig ausgewählte 18- bis 60-jährige SAPALDIA-Population zeigt eine Prävalenz der COPD von 9,1\%, 85\% davon im GOLD-Stadium 1 und wiederum 57\% davon ohne Symptome [2]. Zwischen 1992 und 2003 entwickelten weitere 14,5\% der Untersuchten eine COPD, was einer Inzidenz für diese Erkrankung von 1,3\%/Jahr bei Erwachsenen entspricht. In einer Schweizer Praxisstudie mit über 25000 Spirometrien wurden bei $28 \%$ der untersuchten, über 40-jährigen Rauchenden pathologische Werte gemessen. Gestützt auf diese Untersuchungen gehen wir davon aus, dass bei etwa 400000 Schweizerinnen und Schweizern eine COPD diagnostiziert werden kann.

\section{Ursachen der COPD}

Jeder zweite Raucher wird eine COPD entwickeln, wenn er nicht vorher an einer anderen zigarettenrauchassoziierten Krankheit, einer koronaren Herzkrankheit oder einem Lungenkrebs stirbt [3]. Nur etwa 15 Prozent der Personen mit COPD sind Nichtraucher! In der Schweiz ist neben dem Rauchen eine Tätigkeit als Landwirt der wichtigste Risikofaktor zur Entwicklung einer COPD. Landwirte sind vor allem in ihren Ställen dem Heu- und Strohstaub sowie Dämpfen ausgesetzt, die von den Tieren und deren Ausscheidungen ausgehen. Häufige Atemwegsinfekte durch Bak- 


\section{Abbildung 1}

Klassifizierung des Schweregrades der COPD (aus [1]).

\section{Klassifizierung des Schweregrades der COPD}

\begin{tabular}{|c|c|c|c|c|}
\hline Gefährdet & $\begin{array}{l}\text { Stadium I } \\
\text { Leicht }\end{array}$ & $\begin{array}{l}\text { Stadium II } \\
\text { Mittelschwer }\end{array}$ & $\begin{array}{l}\text { Stadium III } \\
\text { Schwer }\end{array}$ & $\begin{array}{l}\text { Stadium IV } \\
\text { Sehr schwer }\end{array}$ \\
\hline normale Spirometrie & $\begin{array}{l}\mathrm{FEV}_{1} / \mathrm{FVC}<70 \% \\
\mathrm{FEV}_{1} \geq 80 \%\end{array}$ & $\begin{array}{l}\text { FEV }, / \text { FVC }<70 \% \\
\text { FEV }_{1} 50 \%-80 \%\end{array}$ & $\begin{array}{l}\text { FEV }, / F V C<70 \% \\
\text { FEV }, 30 \%-50 \%\end{array}$ & $\begin{array}{l}\text { FEV }, / F V C<70 \% \\
F E V_{1}<30 \%\end{array}$ \\
\hline chronische Symptome & mit oder ohne & mit oder ohne & mit oder ohne & $0 c$ \\
\hline $\begin{array}{l}\text { (Husten, Auswurf): } \\
\text { Exposition gegenüber } \\
\text { Risikofaktoren }\end{array}$ & Symptome & Symptome & Symptome & $\begin{array}{l}\text { Vorliegen einer chron. } \\
\text { Ateminsuffizienz oder } \\
\text { Rechtsherzinsuffizienz }\end{array}$ \\
\hline
\end{tabular}

4 Anthonisen NR, Connett JE, Kiley JP, et al. Effects of smoking intervention and the use of an inhaled anticholinergic bronchodilator on the rate of decline of FEV1: the Lung Health Study. JAMA. 1994;272:1497-505.

5 Anthonisen NR, Skeans MA, Wise RA, et al.The effects of a smoking cessation intervention on 14.5-year mortality. Ann Intern Med. 2005;142:233-9. terien und Viren sind typische Ursachen für Exazerbationen der Erkrankung und beschleunigen das Fortschreiten der Erkrankung. Deshalb werden COPD-Patienten die regelmässige Grippeimpfung und eine Pneumokokkenimpfung empfohlen.

Der einzige bisher gut dokumentierte genetische Risikofaktor für die Entwicklung einer COPD ist der Alpha-1-Antitrypsinmangel. Alpha-1-Antitrypsin ist ein im Blut zirkulierendes, in der Leber gebildetes Eiweiss, das die Lunge vor inhalierbaren Schadstoffen bzw. vor deren dadurch ausgelösten Entzündung schützen kann. Diese Erbkrankheit kann einfach durch die Messung der Konzentration von Alpha-1-Antitrypsin im Blut diagnostiziert werden. Diese Untersuchung soll bei allen Patienten, die an einer besonderen Manifestation der COPD leiden (Alter < 45 Jahren; Nichtraucher) veranlasst werden. Es gibt Hinweise dafür, dass bei gewissen Patienten durch intravenöse Substitution von Alpha-1-Antitrypsin der Verlauf der Lungenerkrankung gebremst werden kann. Diese Behandlung ist teuer und muss lebenslänglich erfolgen. Weitere Erkrankungen, die sich funktionell wie eine COPD mit einer weitgehend fixierten Obstruktion der Atemwege manifestieren, sind Zustände nach durchgemachter Bronchiolitis sowie rezidivierende Infekte der grossen und kleinen Atemwege bei Zystischer Fibrose, Immunglobulinmangel und Dysmotilen Zilien (z. B. beim vom Schweizer Arzt Kartagener 1933 beschriebenen und nach ihm benannten Syndrom).

\section{COPD ist heute behandelbar}

Das frühere Stigma der COPD einer hoffnungslosen Krankheit ist überholt: Die amerikanische Lung Health Study hat gezeigt, dass bei Rauchern, motiviert durch eine pathologische Lungenfunktionsmessung, durch eine erfolgreiche Raucherentwöhnung der weitere $\mathrm{FEV}_{1}$-Abfall gebremst werden kann [4] und die Mortalität signifikant gesenkt werden kann [5]. Moderne Bronchodilatatoren, Antibiotika und Kortikosteroide können Häufigkeit und Schweregrad von Exazerbationen günstig beeinflussen. Bei Auftreten von limitierenden Symptomen sind die Patientenschulung und ein ärztlich überwachtes Körpertraining für den Behandlungserfolg entscheidend. Im Spätstadium ist oft eine Sauerstoffheimtherapie, selten eine Lungenvolumenreduktionsoperation oder Lungentransplantation nötig. Wie bei Krebskranken wird die Besprechung von möglichen Massnahmen am Lebensende zu einer weiteren schwierigen ärztlichen Aufgabe.

\section{Welt-COPD-Tag 19. November 2008}

Die Lungenliga hilft. Sie setzt sich für die Prävention von Lungen- und Atemwegserkrankungen ein. Die Fachkräfte der Lungenliga beraten und begleiten Betroffene. In enger Zusammenarbeit mit der Ärztin oder dem Arzt stellt die Lungenliga die für die Therapie erforderlichen Atemhilfsgeräte bereit. Sie bietet Rauchstoppberatungen und-kurse an. Die Lungenliga bietet einen Onlinerisikotest auf www.copd.Iungenliga.ch an. Interessierte und Risikopersonen können ihr COPD-Risiko mit einem einfachen Test abklären. Sie erfahren, ob sich für sie ein Arztbesuch empfiehlt. Das Onlineforum auf www.copd.lungenliga.ch bietet Betroffenen und Interessierten eine Plattform, um medizinischen Rat einzuholen und Erfahrungen auszutauschen. Es wird vom 10. bis 28 . November 2008 vom Lungenspezialist Prof. Dr. Roland Keller moderiert. Die leicht verständliche Broschüre «COPD Chronisch obstruktive Lungenerkrankung Symptome - Diagnose - Behandlung» informiert Betroffene, Angehörige und Interessierte. Sie kann kostenlos in Deutsch, Französisch und Italienisch über www.publikationen. lungenliga.ch, per E-Mail bei info@lung.ch oder per Telefon unter 0313782050 bestellt werden.

Lungenliga Schweiz, Südbahnhofstrasse 14c, 3000 Bern 14, Tel. 03137820 50, Fax 031 37820 51, E-Mail: info@lung.ch. Internet: www.lungenliga.ch 